\title{
Insulin receptor substrate- 1 and dishevelled 2 are negatively regulated by microRNA-144 and inhibit nasopharyngeal carcinoma cell malignancy
}

\author{
XUEMEI AN $^{1 *}$, YUNLAN JIANG $^{1 *}$, DEFENG CHEN $^{1}$ and JIANJUN CHEN ${ }^{2}$ \\ ${ }^{1}$ Hospital of Chengdu University of Traditional Chinese Medicine, Chengdu, Sichuan 410000; \\ ${ }^{2}$ Department of Ear, Nose and Throat, The First People's Hospital of Neijiang, Neijiang, Sichuan 641000, P.R. China
}

Received April 19, 2020; Accepted April 16, 2021

DOI: $10.3892 / \mathrm{etm} .2021 .10738$

\begin{abstract}
Insulin receptor substrate-1 (IRS-1) is reported to play a critical role in the development, progression, invasion and metastasis of several types of tumors and is abnormally expressed in nasopharyngeal carcinoma (NPC). Although IRS-1 is predicted to be targeted by microRNA (miR)-144, the biological roles and potential mechanisms of miR-144 in NPC remain unclear. In the present study, the expression levels of miR-144 and IRS-1 in several NPC cell lines were first examined, and found that they were negatively correlated. Following the introduction of the miR-144 mimic, IRS-1 was downregulated at the protein level without affecting the mRNA level. The Cell Counting Kit-8 assay showed that the miR-144 mimic and siRNA targeting IRS-1 mRNA significantly decreased cell proliferation by arresting the cell cycle at the $\mathrm{G}_{1} / \mathrm{G}_{0}$ phase. The malignant behaviours of NPC cell lines, including migration, invasion and tumour formation in soft agar, were then analyzed after regulating miR-144 levels; as expected, the results showed that both the miR-144 mimic and siIRS-1 decreased these malignant behaviours. Furthermore, the downregulation of IRS-1 by miR-144 decreased the expression level of dishevelled 2 (Dvl2) protein without affecting its mRNA level, and Dvl2 overexpression abolished the inhibitory effect of the miR-144 mimic in NPC, indicating that miR-144 potentially regulates NPC by indirectly regulating Dvl2. Taken together, the present study results suggest that miR-144 acts as a tumour suppressor in NPC cell lines by regulating IRS-1 and Dvl2, which indicates that it is a potential therapeutic target for NPC treatment.
\end{abstract}

Correspondence to: Dr Jianjun Chen, Department of Ear, Nose and Throat, The First People's Hospital of Neijiang, 31 Jiaotong Road, Tuojiang Street, Neijiang, Sichuan 641000, P.R. China

E-mail: chengjianjun928@163.com

*Contributed equally

Key words: insulin receptor substrate-1, nasopharyngeal carcinoma, cell proliferation, dishevelled 2, microRNA-144

\section{Introduction}

Nasopharyngeal carcinoma (NPC) is a head and neck malignant tumour derived from nasopharyngeal epithelium and has a high incidence rate, with $>20$ cases per 100,000 individuals in southern China (1). Several factors contribute to NPC occurrence and development, including Epstein-Barr virus (EBV) infection, chemical carcinogens, genetic variation and spontaneous mutation (2). NPC is characterized by metastases via both lymph and blood vessels at the early stage of the disease (3), and subsequently induces the development of distant metastases to the bone, lung and liver, which are major causes of treatment failure after different treatments (4). Although much effort has been devoted, the mechanisms that regulate NPC malignant behaviours, including proliferation, migration, invasion and colony formation, remain poorly understood.

Small non-coding microRNAs (miRNAs/miRs) are a group of small, non-coding RNA molecules that play critical roles in regulating tumorigenesis processes. Moreover, accumulating evidence has demonstrated that abnormalities in the expression profiles of miRNAs are a hallmark of tumorigenesis and development (5). By acting as tumour promotors or inhibitors, miRNAs tightly regulate the occurrence, development and progression of cancer (6). miR-144 is an miRNA identified as a tumour regulator in several types of cancer, including lung cancer, nasopharyngeal carcinoma, hepatocellular carcinoma and clear cell renal cell carcinoma (7-10). However, miR-144 has been reported to act as either an oncogene or tumour suppressor in different types of cells, which has raised an interesting study topic on its regulatory role in NPCs.

Insulin receptor substrate (IRS) proteins are a group of signalling intermediates downstream of cytoplasmic molecules that play critical roles in several diseases, including cancer (11). One member of IRS, termed insulin receptor substrate-1 (IRS-1), was reported to be associated with tumour initiation and progression. IRS-1, activated by insulin/insulin-like growth factor I receptors, induces the transduction of intracellular cascades, including activating PI3K-AKT and Ras-MAPK pathways (12). As IRS-1 regulates several basal processes, including inhibiting autophagy and decreasing oxidative stress-induced apoptosis, the 
overexpression of IRS-1 promotes malignant behaviours in several cancer cells $(13,14)$. A study reported that IRS-1 is downregulated by miR-145 and leads to the inhibition of proliferation in colon cancer cells (15). Wu et al (16) reported that, in laryngeal squamous cell carcinoma, miR-144 suppresses the growth and metastasis by targeting IRS1, indicating that miRNAs, including miR-144, may regulate malignant behaviours in cancer by regulating IRS-1.

Dishevelled (Dvl)2 is a cytoplasm-nucleus shuttling protein and thus tightly regulates Wnt signaling (17), which is regulated by a variety of factors, including ubiquitin-proteasome and autophagy-lysosomal pathways $(18,19)$. Zhang et al (18) reported that Dvl2 is bound by GABARAPL1, a member of the ATG8 family, and results in degradation through autophagy. Further research also revealed that IRS-1 interacts directly with Dvl2, and overexpression of IRS-1 increased the protein level of Dvl2 by inhibiting ubiquitination and subsequent degradation (20). Subsequently, Dvl2 stabilized by IRS-1 promotes Wnt/ $\beta$-catenin signalling and thus promotes epithelial-mesenchymal transition (EMT) and cell proliferation in response to Wnt stimulation (20). These results indicate that miRNAs may regulate Dvl2 signalling by post-transcriptional regulation of IRS-1 and modifying physiological processes.

The present study focused on the biological functions and underlying molecular mechanisms of miR-144 via its regulation of IRS-1 and subsequent regulation of malignant behaviors of NPCs.

\section{Materials and methods}

Cell culture. Nasopharyngeal carcinoma cell lines NPC-TW01 and SUNE1 were bought from The Cell Bank of Type Culture Collection of The Chinese Academy of Sciences. All cells were maintained in RPMI-1640 medium (Thermo Fisher Scientific, Inc.) supplemented with $10 \%$ fetal bovine serum (FBS; Thermo Fisher Scientific, Inc.) in a humidified atmosphere containing $5 \% \mathrm{CO}_{2}$ at $37^{\circ}$. Cells were passaged every three days.

Reverse transcription-quantitative $(R T-q) P C R$. Total RNA was isolated using TRIzol ${ }^{\circledR}$ according to manufacturer's instruction and was reverse transcribed into complementary DNA using Bio-Rad I Script cDNA Synthesis System (Bio-Rad Laboratories, Inc.) at $42^{\circ} \mathrm{C}$ for $60 \mathrm{~min}$ and denatured at $80^{\circ} \mathrm{C}$ for $10 \mathrm{~min}$. SYBR-Green PCR Master mix (Thermo Fisher Scientific, Inc.) was then employed for qPCR according to manufacturer's instruction. The primers used were as follows: IRS-1 forward, 5'-CAAGACCATCAGCTTCGTGA-3'; IRS-1 reverse, 5'-AGA GTCATCCACCTGCATCC-3'; Dvl2 forward, 5'-GCCTATCCA GGTTCCTCCTC-3'; Dvl2 reverse, 5'-AGAGCCAGTCAACC ACATCC-3'; $\beta$-actin forward, 5'-CATGTACGTTGCTATCCA GGC-3'; and $\beta$-actin reverse, 5'-CTCCTTAATGTCACGCAC GAT-3'. For quantitative detection of miRNAs, total RNA was revere transcribed using All-in-One ${ }^{\text {TM }}$ miRNA qRT-PCR Detection System (Guangzhou RiboBio Co., Ltd.) following the manufacturer's instructions. Primer for miR-144 (cat. no. HmiRQP0910) and RNU6-2 (cat. no. HmiRQP9001) were bought from Guangzhou RiboBio Co., Ltd. Relative quantification of mRNA and miRNA expression were calculated by using the $2^{-\Delta \Delta \mathrm{Cq}}$ method (21). All RT-qPCR reactions were performed in triplicates.
Western blotting. The cells were lysed using RIPPA buffer (Sigma-Aldrich; Merck KGaA) and total protein concentration was quantified using the BCA protein assay kit (Sigma-Aldrich; Merck KGaA) following the manufacturer's instructions. Total protein $(20 \mu \mathrm{g})$ was separated by $10 \%$ sodium dodecyl sulfate polyacrylamide gel electrophoresis, transferred to PVDF membrane and blocked with TBS-Tween $(0.1 \%)$ containing 5\% bovine serum albumin (Sigma-Aldrich; Merck $\mathrm{KGaA}$ ) at room temperature for $30 \mathrm{~min}$, and probed with the specific primary antibodies at room temperature for $60 \mathrm{~min}$. Anti-IRS-1 antibody (cat. no. ab52167), anti-Dvl2 antibody (cat. no. ab22616) and anti- $\beta$-actin antibody (cat. no. ab8226) were all bought from Abcam, and diluted in 1:2,000 before use. Then, the blot was incubated with specific horseradish peroxidase (HRP)-conjugated secondary antibodies (cat. nos. ab7090 or ab97040; Abcam) at dilution of 1:1,000, and the immunoreactive bands were imaged using enhanced chemiluminescence (Thermo Fisher Scientific, Inc.). The blots were analyzed using ImageJ software (version-2.0; National Institutes of Health).

Cell Counting Kit-8 (CCK-8) assay. For cell proliferation assay, $5 \times 10^{3}$ cells were seeded into 96 -well plates and were analyzed at the indicated time points (24, 48, 72,96 and $120 \mathrm{~h})$. Then, $10 \mu \mathrm{l}$ CCK-8 reagent (Sigma-Aldrich; Merck KGaA) was added into each well at $37^{\circ} \mathrm{C}$ for another 2 -h incubation. The OD470 $\mathrm{nm}$ value was determined by a microplate reader (Synergy 2 Multi-Mode Microplate Reader; BioTek Instruments, Inc.).

Transfection. PcDNA3.1-Dvl2 plasmid was synthesized by Invitrogen (Thermo Fisher Scientific, Inc.), and empty vector pcDNA3.1 was employed as negative control. Small interfering (si) RNA target to IRS-1 (5'-CAAUGAGUGUGCAUAAACUUC-3'), miR-144 mimic (5'-UACAGUAUAGAUGAUGUACU-3'), miR-144 inhibitor (5'-UACAGUAUAGAUGAUGUACU-3') and corresponding negative controls were bought from Guangzhou RiboBio Co., Ltd. Lipofectamine ${ }^{\circledR} 2000$ (Thermo Fisher Scientific, Inc.) was employed for transfection. For plasmid transfection, $1.6 \mu \mathrm{g}$ of plasmid was used. For mimic, inhibitor and siRNA transfection, $50 \mu \mathrm{M}$ of sample was employed following the manufacturer's instruction. After transfection $(4 \mathrm{~h})$, the cells were employed for further analyses.

Cell cycle analysis. The cells of interest were trypsinized and suspended with PBS, and then pelleted. After discarding the supernatant, cells were resuspended and fixed with ice-cold $70 \%$ ethanol. After $4 \mathrm{~h}$, cells were pelleted and washed with ice-cold PBS, and suspended with $5 \mu \mathrm{g} / \mathrm{ml}$ propidium iodide (PI; Sigma-Aldrich; Merck KGaA) for 10 min in darkness at room temperature. Then, stained cells were analyzed using 3 laser Navios flow cytometers (Beckman Coulter, Inc.) and results were analyzed using FlowJo software (FlowJo LLC; version 9.7.4).

Scratch assay. When the cells reached $95 \%$ confluence, cells were scratched with a sterile plastic micropipette tip. After wounding, plates were washed three times with ice-cold PBS, and RIPM-1640 without FBS was added for 24-h incubation. After culturing for $24 \mathrm{~h}$, images were captured by an inverted 

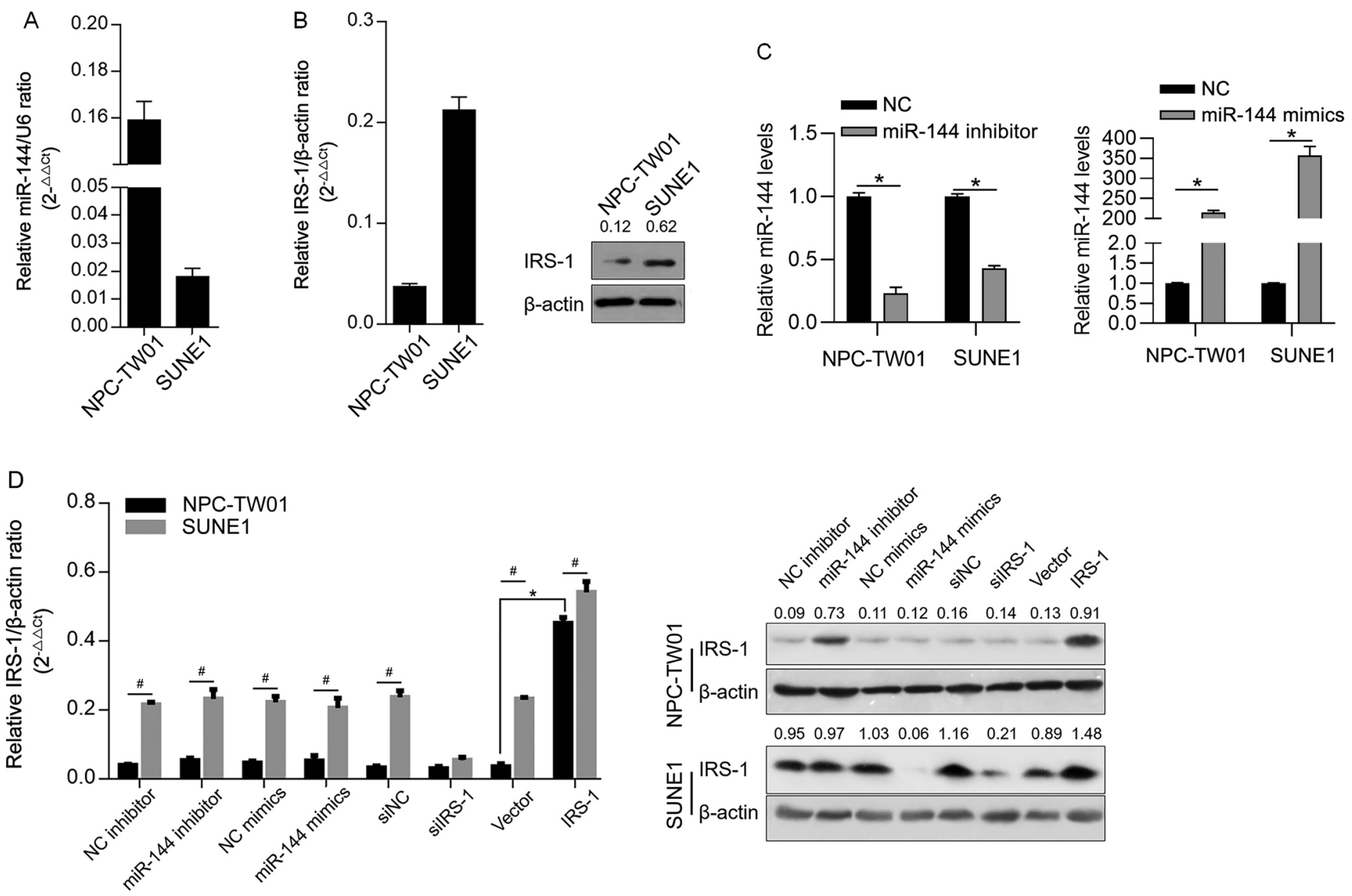

Figure 1. Expression levels of miR-144 and IRS-1 are negatively correlated in NPC cells lines. (A) miR-144 levels were measured and normalized to U6 in NPC cell lines, including NPC-TW01 and SUNE1. (B) IRS-1 mRNA (left panel) and protein (right panel) levels were measured. (C) Reverse transcription-quantitative PCR was performed to detect the efficacy of miR-144 inhibition or upregulation. (D) After introducing miR-144 inhibitor, miR-144 mimic, siIRS-1 or IRS-1, IRS-1 mRNA (left panel) and protein (right panel) levels were measured. " $\mathrm{P}<0.05$ vs. vector group; $\mathrm{P}<0.05$ vs. untreated group. miR, microRNA; NPC, nasopharyngeal carcinoma; IRS-1, insulin receptor substrate-1; si, small interfering RNA; NC, negative control.

fluorescence microscope (Nikon Corporation; magnification, $\mathrm{x} 40$ ) and the wound healing ability of each cell line was analyzed. Distances between the edges of the scratch were measured using ImageJ software (version-2.0; National Institutes of Health) to quantitatively evaluate cell migration. Cell migration was calculated according to the following formula: Cell migration ratio $=100 \times(0 \mathrm{~h}$ scratch width- $24 \mathrm{~h}$ scratch width) $/ 0 \mathrm{~h}$ scratch width.

Transwell assay. In order to analyze invasion capacity, $1 \times 10^{4}$ cells were suspended and plated into the upper chamber of Transwell inserts ( $8-\mu \mathrm{m}$ pore size; Corning Inc.), coated with 0.3\% Matrigel (Sigma-Aldrich; Merck KGaA) in RPMI-1640 medium without FBS addition, and maintained in an incubator at $37^{\circ} \mathrm{C}$. In the lower chamber, $500 \mu 1 \mathrm{RPMI}-1640$ medium supplemented with $10 \%$ FBS was added. After 24-h incubation, the invaded cells were fixed with $4 \%$ paraformaldehyde at room temperature for $10 \mathrm{~min}$ and stained with $0.25 \%$ crystal violet (Sigma-Aldrich; Merck KGaA) at room temperature for $10 \mathrm{~min}$. Invaded cells were then imaged under a X71 (U-RFL-T) fluorescence microscope (Olympus Corporation; magnification, $\mathrm{x} 40$ ).

Colony formation in soft agar. Collected cells were counted and resuspended in $0.3 \%$ low-melting soft agar in RPMI-1640 containing $10 \%$ FBS, then plated onto $0.6 \%$ solidified agar in RPMI-1640 containing 10\% FBS in 6-well plates $\left(5 \times 10^{3}\right.$ cells/well). The samples were maintained at $37^{\circ} \mathrm{C}$ for 3 weeks. Colonies containing 50 cells or more were counted under a X71 (U-RFL-T) fluorescence microscope (Olympus Corporation; magnification, $\mathrm{x} 40$ ).

Statistical analysis. In the present study, all data are presented as the mean $\pm \mathrm{SD}$. One-way analysis of variance (ANOVA) was performed to compare multiple groups with one variable followed by Tukey's post-hoc test. Significance between two groups was calculated by Student's t-test. $\mathrm{P}<0.05$ was considered to indicate a statistically significant difference.

\section{Results}

Expression of miR-144 post-transcriptionally regulates IRS-1 in NPC cells. In order to determine the expression level of miR-144 in NPC cell lines, RT-qPCR was performed to detect miR-144 level normalized to U6 RNA. It was observed that miR-144 was detected in all tested NPC cell lines with different expression profiles (Fig. 1A). The protein levels of IRS-1 in the cell lines were then detected by semi-quantitative western blotting. As shown in Fig. 1B, IRS-1 was detected in all these cell lines. By considering that the expression 

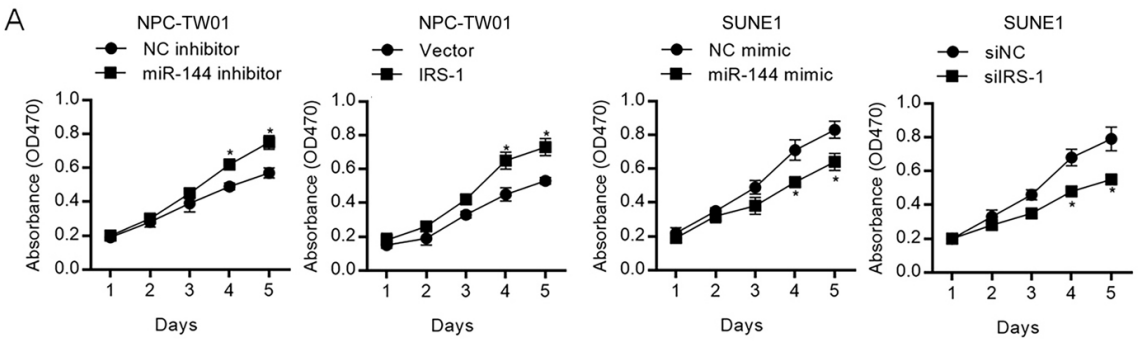

B
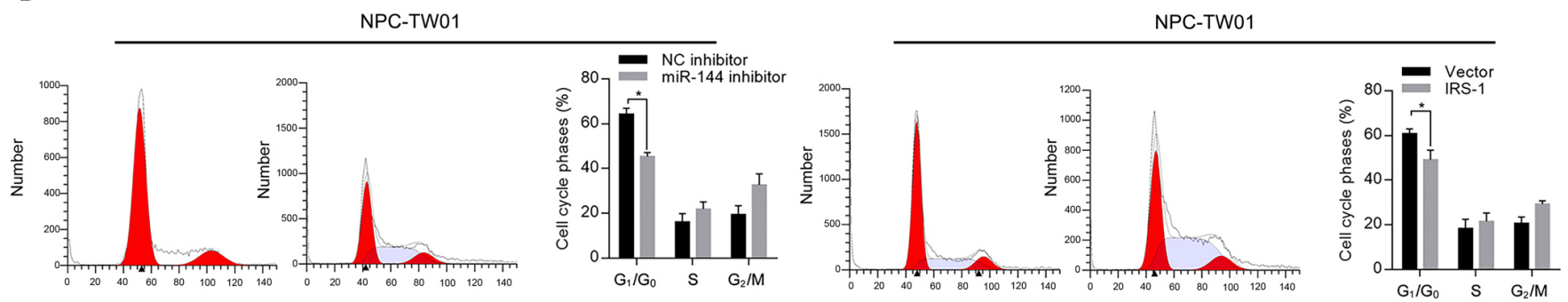

NPC-TW01
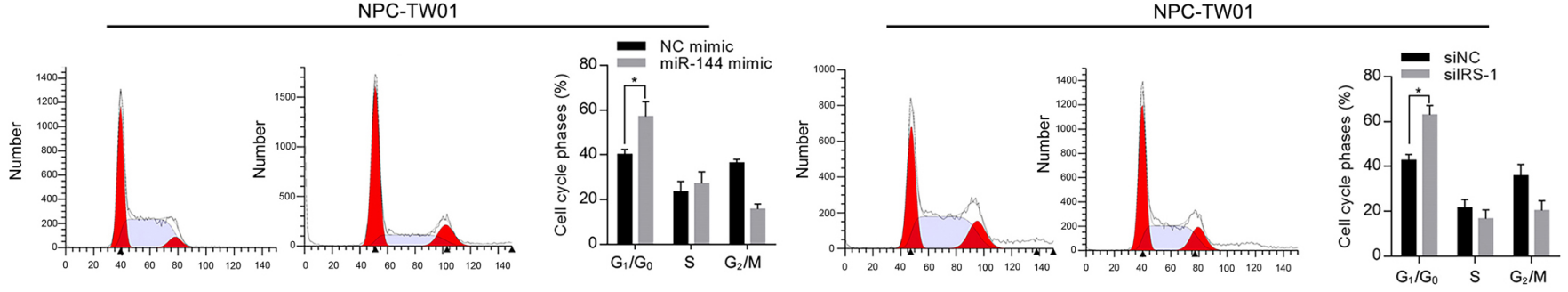

Figure 2. miR-144 inhibits cell proliferation potentially via targeting IRS-1 in nasopharyngeal carcinoma cells. (A) After introducing miR-144 inhibitor, miR-144 mimic, siIRS-1 or IRS-1, cell viability was measured by performing Cell Counting Kit- 8 assay. * P<0.05 vs. control group. (B) The aforementioned cells were stained with PI and the proportion of cell cycle phases of the cells were analyzed by performing flow cytometry. ${ }^{*} \mathrm{P}<0.05$ vs. control group. miR, microRNA; IRS-1, insulin receptor substrate 1; si, small interfering RNA; NC, negative control.

levels of miR-144 and IRS-1 were contrasting in NPC-TW01 and SUNE1 cells, these two cells were selected for further analysis. To confirm whether miR-144 post-transcriptionally regulates IRS-1, miR-144 inhibitor, miR-144 mimic, siIRS-1 or IRS-1 were efficiently transfected, confirmed by RT-qPCR (Fig. 1C), and IRS-1 protein was measured $48 \mathrm{~h}$ later. As shown in Fig. 1D, without affecting IRS-1 mRNA, the introduction of miR-144 inhibitor increased IRS-1 protein, whereas the miR-144 mimic downregulated the expression level of IRS-1 protein, which was similar to the observation following siIRS-1 transfection. Briefly, miR-144 post-transcriptionally regulates IRS-1.

miR-144 inhibits cell proliferation by targeting IRS-1 in NPC cells. In order to evaluate the effect of miR-144 on proliferation, the expression level of miR-144 in NPC-TW01 or SUNE1 was modified by introducing miR-144 inhibitor or miR-144 mimic. CCK-8 cell viability assay results presented that, in NPC-TW01 cells, both the inhibition of miR-144 and overexpression of IRS-1 significantly promoted cell proliferation, and in SUNE1 cells, both the introduction of miR-144 mimic and knockdown of IRS-1 significantly inhibited cell proliferation (Fig. 2A). The opposite effect of miR-144 and IRS-1 on cell proliferation indicated that miR-144 potentially regulates cell proliferation via regulating IRS-1. To further confirm the regulation of proliferation by miR-144, cell cycle was then assessed by flow cytometry. As shown in Fig. 2B, miR-144 expression significantly blocked the cell cycle at the $G_{1} / G_{0}$ phase, which is in contrast to the observation in the presence of IRS-1.

miR-144 decreases malignant behaviors in NPC cells. In order to further access whether miR-144 is relevant in other malignant behaviors of NPC cells, cell migration, invasion and tumor formation in soft agar were examined after modification of miR-144 or IRS-1. As shown in Fig. 3A-C, in NPC-TW01 cells, inhibition of miR-144 and overexpression of IRS-1 promoted migration, invasion and colony formation in soft agar, and in SUNE1, the introduction of miR-144 mimic and knockdown of IRS-1 obviously inhibited migration, invasion and colony formation in soft agar. All these observations are consistent with the effect of miR-144 on proliferation, indicating that miR-144 exerted inhibitory effects not only on proliferation.

miR-144 acts as a tumor suppressor potentially via downregulating Dvl2 indirectly. Accordingly, IRS-1 stabilizes Dvl2 and thus promotes malignant behaviors in cancer cells (20). Thus, whether miR-144 regulates Dvl2 protein level was further confirmed. As shown in Fig. 4A, miR-144 negatively regulated IRS-1 and Dvl2 without disturbing Dvl2 mRNA, indicating that miR-144 may regulate the proliferation of NPC cells via indirectly regulating Dvl2. Thus, Dvl2 was modified in NPC-TW01 and SUNE1 cells by efficiently introducing 
A
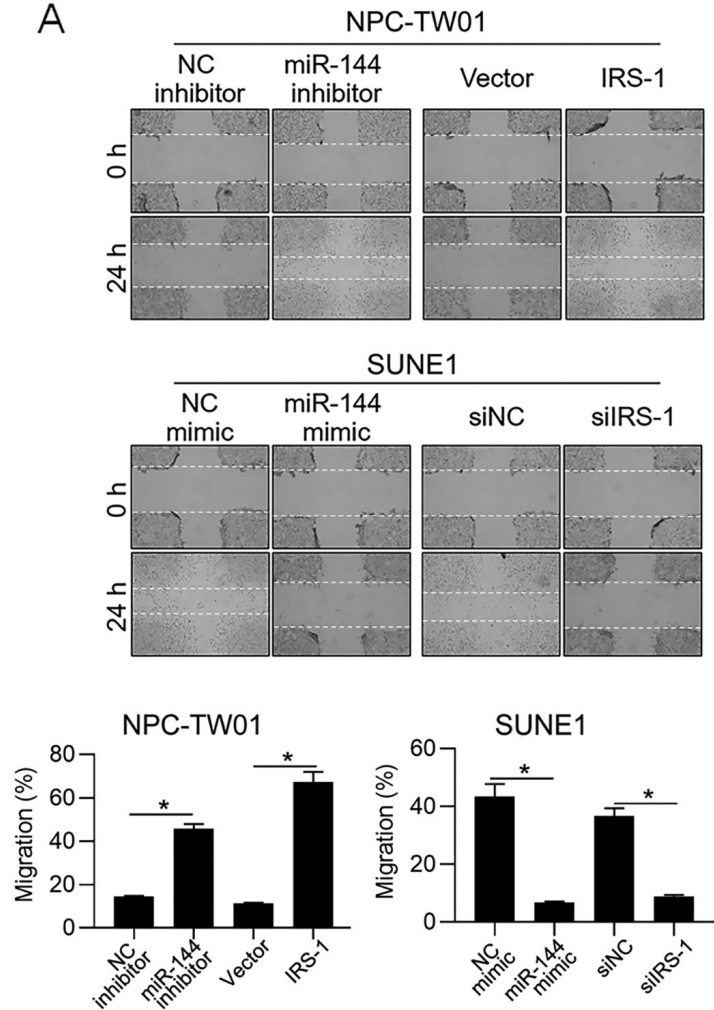

SUNE1

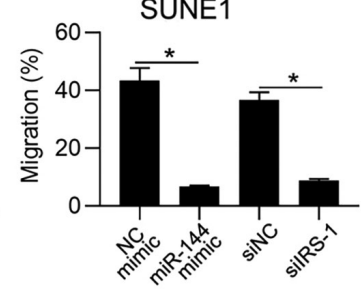

B
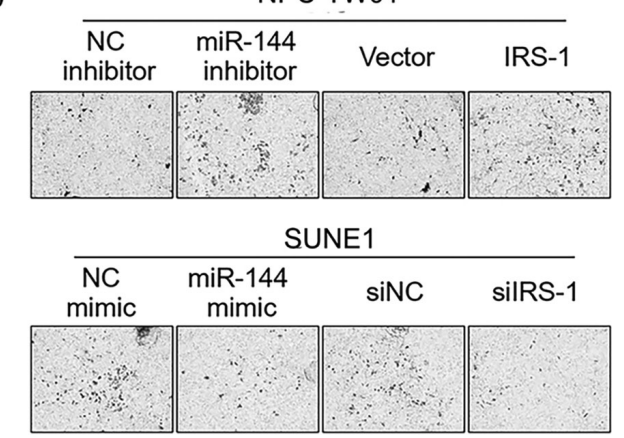

C
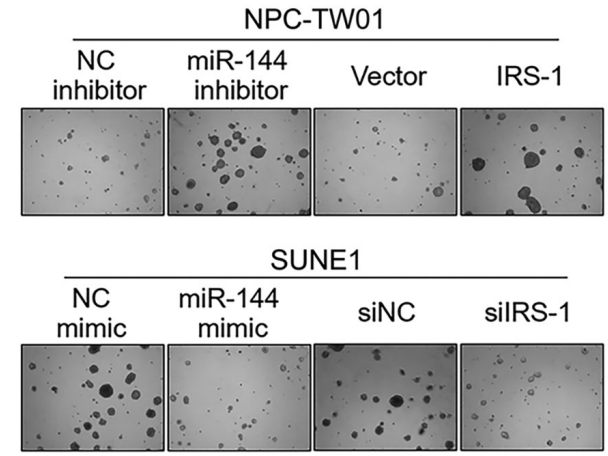

Figure 3. Presence of miR-144 tightly regulates malignant behaviors in nasopharyngeal carcinoma cells potentially via regulating IRS-1. After introducing miR-144 inhibitor, miR-144 mimic, siIRS-1 or IRS-1, (A) cell migration, (B) invasion and (C) tumor formation in soft agar were assessed. * $<<0.05$ vs. vector group. miR, microRNA; IRS-1, insulin receptor substrate 1; si, small interfering RNA; NC, negative control.
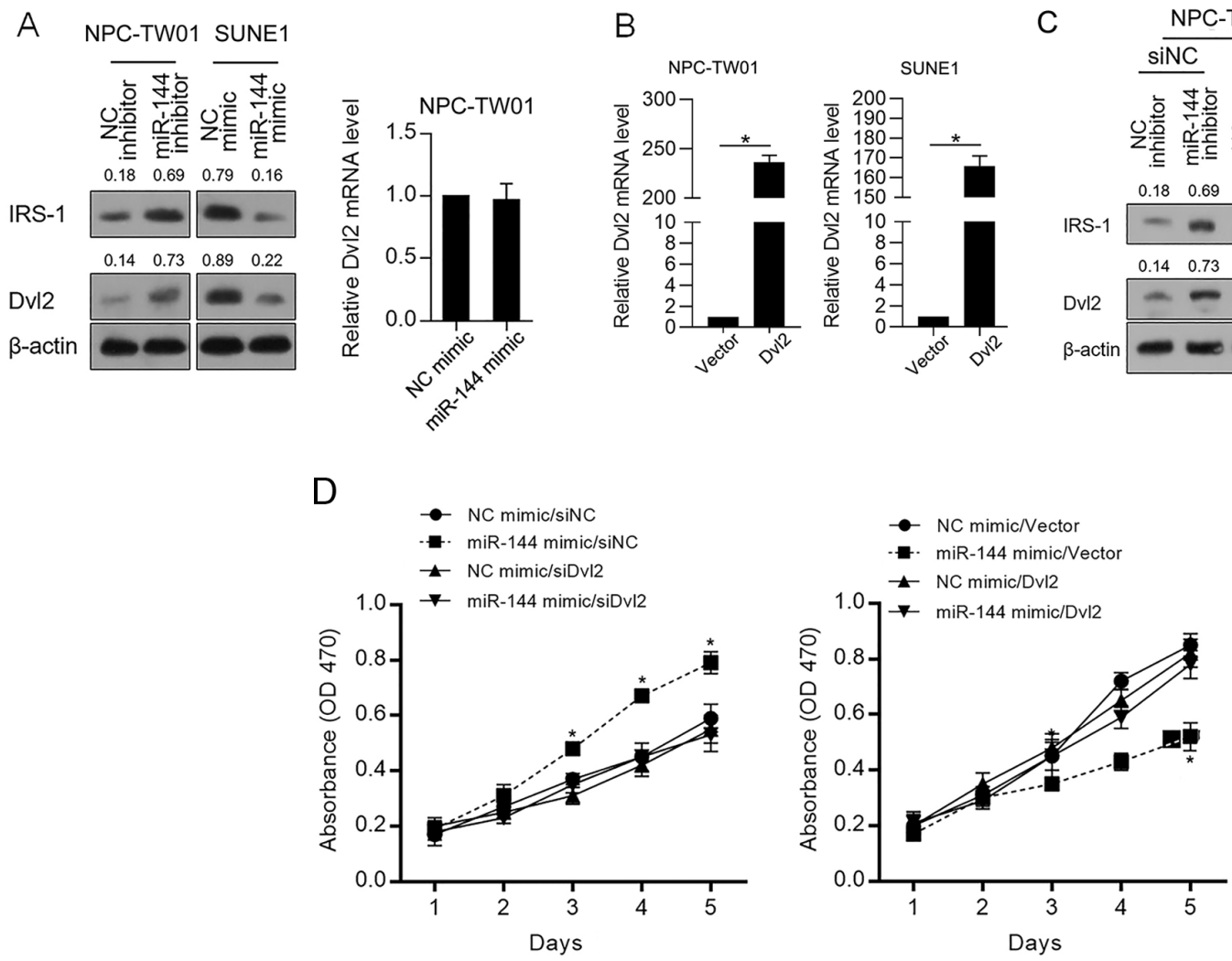

Figure 4. miR-144 inhibits cell proliferation via indirectly regulating Dvl2. (A) miR-144 regulates Dvl2 protein level without disturbing its mRNA level. (B) After transfecting the Dvl2-coding vector, reverse transcription-quantitative PCR was performed to detect Dvl mRNA level. ${ }^{*} \mathrm{P}<0.05$ vs. vector group. (C) The knockdown efficacy of siRNA target to Dvl2 is confirmed by semi-quantitative western blotting. (D) The cell proliferation was measured after introducing miR-144 mimic, siDvl2, Dvl2 together or individually in nasopharyngeal carcinoma cells. "P<0.05 vs. control group. miR, microRNA; Dvl2, dishevelled 2; IRS-1, insulin receptor substrate 1; si/siRNA, small interfering RNA; NC, negative control. 
a Dvl2-coding vector (Fig. 4B). As shown in Fig. 4C, Dvl2 protein expression was successfully modified despite the level of IRS-1. Then, cell proliferation was analyzed. In Fig. 4D, it was observed that the inhibitory effect of miR-144 on proliferation was significantly reversed by overexpression of Dvl2, demonstrating that the inhibitory effect of miR-144 on proliferation depends on the decrease in Dvl2.

\section{Discussion}

Accumulating evidence indicates that miRNAs exert significant roles in the pathogenesis of NPC by regulating tumour cell proliferation, metastasis, invasion and chemosensitivity (22). For example, Qiu et al (23) reported that in NPC cells, miR-29a/b targeted the COL3A1 and SPARC genes and thus enhanced cell migration, invasion and proliferation. Verhoeven et al (24) reported that miRNA and long non-coding RNA are regulated by high levels of EBV, and overexpressed miRNAs then promote malignant behaviors in NPC cells. It has also been reported that miR-24 promotes DNA methylation and then acts as a radiosensitizer in NPC cells. Further analysis revealed that miR-24 is downregulated in NPC tissues compared with adjacent tissues (25). The present study found that miR-144 expression is differentially expressed in different NPC cell lines, and its expression is negatively correlated with the IRS-1 level, which was reported to be a target that is downregulated post-transcriptionally. Overexpression of the miR-144 mimic remarkably decreased the malignant behaviors of NPCs, including proliferation, migration, invasion and tumour formation in soft agar. Conversely, the downregulation of miR-144, by introducing a specific inhibitor, promoted the aforementioned malignant behaviors. Zhang et al (8) reported that miR-144 promoted NPC malignancy by inhibiting PTEN expression, which is in contradiction with the present study results. The difference may be due to the different expression patterns of PTEN in different NPC cell lines, and it is worth investigating the roles of PTEN in additional NPC cell lines.

Dvl2 is mainly involved in the regulation of $\mathrm{Wnt} / \beta$-catenin signaling (17). In recent research, Dvl2 was found to directly bind to the IRS-1 protein (20). IRS-1 binds to Dvl2 with or without insulin stimulation, and its overexpression increases the protein level of Dvl2, thus promoting canonical Wnt signalling and increasing the expression of CYCLIN D1 and C-MYC, which are recognized as oncogenes (20). Further research showed that the binding of IRS-1 to Dvl2 decreased ubiquitination and induced the stabilization of Dvl2. According to the present study results and consistent with previous findings, downregulation of IRS-1 by miR-144 introduction decreased the protein level of Dvl2. Although the binding of IRS-1 and Dvl2 was not investigated further, the presented results also demonstrated that the regulation of IRS-1 by miR-144 negatively regulated Dvl2. Moreover, the inhibitory effect of the miR-144 mimic on proliferation was significantly abolished by the exogenous expression of Dvl2, indicating that the regulatory effects of miR-144 are potentially, at least in part, dependent on the regulation of Dvl2.

IRS has been identified as a key player in the regulation of cell proliferation and epithelial mesenchymal transition (EMT), which is characterized by the expression levels of E-cadherin and N-cadherin. It has been reported that the overexpression of IRS-1 regulates the expression of E-cadherin during EMT $(26,27)$ and cyclinD1 during cell proliferation (28). Although the hallmarks of EMT or the effects of miR-144 on EMT were not detected, by performing scratch and transwell assays, it was confirmed that miR-144 inhibited the migration and invasion of NPC cells, which was similar to the effect of IRS-1 knockdown being reversed by the introduction of a miR-144 inhibitor, indicating that miR-144 potentially regulates EMT. By considering that miR-144 post-transcriptionally regulates IRS-1 by direct binding (29), dual-luciferase reporter gene assay was not performed to prove that miR-144 post-transcriptionally regulates IRS-1, which is a limitation of the present study.

In conclusion, the present study findings show that miR-144 regulates IRS-1 and Dvl2. miR-144 inhibited the malignant behavior of NPC cells, including proliferation, migration, invasion and tumour formation, mainly via indirect regulation of Dvl2. The findings improve the understanding of the molecular mechanisms of miR-144 in the physiological processes of NPC cells. In future research, it is worth confirming whether miR-144 regulates EMT and the Wnt/ $\beta$-catenin signaling pathway.

\section{Acknowledgements}

The authors would like to thank Professor Tao Hong (Sichuan University, Chengdu, China) for language editing.

\section{Funding}

This work was supported by Scientific Fund of the First People's Hospital of Neijiang (grant no. 18ZF0399).

\section{Availability of data and materials}

The datasets generated and/or analyzed during the current study are available from the corresponding author on reasonable request.

\section{Authors' contributions}

XA and JC designed the experiments, assessed the raw data and are responsible for confirming the authenticity of the data. XA, YJ and DC performed cell culture-associated experiments. DC and JC are responsible for data collection and performed statistical analysis. All authors read and approved the final manuscript.

\section{Ethics approval and consent to participate}

Not applicable.

\section{Patient consent for publication}

Not applicable.

\section{Competing interests}

The authors declare that they have no competing interests. 


\section{References}

1. Cho WC: Nasopharyngeal carcinoma: Molecular biomarker discovery and progress. Mol Cancer : 1, 2007.

2. Chou J, Lin YC, Kim J, You L, Xu Z, He B and Jablons DM: Nasopharyngeal carcinoma - review of the molecular mechanisms of tumorigenesis. Head Neck 30: 946-963, 2008.

3. Blanchard P,Lee A, Marguet S, Leclercq J, Ng WT, Ma J, Chan AT, Huang PY, Benhamou E, Zhu G, et al; MAC-NPC Collaborative Group: Chemotherapy and radiotherapy in nasopharyngeal carcinoma: An update of the MAC-NPC meta-analysis. Lancet Oncol 16: 645-655, 2015

4. Lai SZ, Li WF, Chen L, Luo W, Chen YY,Liu LZ, Sun Y, Lin AH, Liu MZ and Ma J: How does intensity-modulated radiotherapy versus conventional two-dimensional radiotherapy influence the treatment results in nasopharyngeal carcinoma patients? Int J Radiat Oncol Biol Phys 80: 661-668, 2011.

5. Shukla GC, Singh J and Barik S: MicroRNAs: Processing, maturation, target recognition and regulatory functions. Mol Cell Pharmacol 3: 83-92, 2011.

6. Iorio MV and Croce CM: Causes and consequences of microRNA dysregulation. Cancer J 18: 215-222, 2012.

7. Chen S, Li P, Li J, Wang Y, Du Y, Chen X, Zang W, Wang H, Chu H, Zhao G, et al: MiR-144 inhibits proliferation and induces apoptosis and autophagy in lung cancer cells by targeting TIGAR. Cell Physiol Biochem 35: 997-1007, 2015.

8. Zhang LY, Ho-Fun Lee V, Wong AM, Kwong DL, Zhu YH, Dong SS, Kong KL, Chen J, Tsao SW, Guan XY, et al: MicroRNA-144 promotes cell proliferation, migration and invasion in nasopharyngeal carcinoma through repression of PTEN. Carcinogenesis 34: 454-463, 2013.

9. BaoH,Li X,LiH,Xing H, Xu B,Zhang X and LiuZ: MicroRNA-144 inhibits hepatocellular carcinoma cell proliferation, invasion and migration by targeting ZFX. J Biosci 42: 103-111, 2017.

10. Liu F, Chen N, Xiao R, Wang W and Pan Z: miR-144-3p serves as a tumor suppressor for renal cell carcinoma and inhibits its invasion and metastasis by targeting MAP3K8. Biochem Biophys Res Commun 480: 87-93, 2016.

11. Mardilovich K, Pankratz SL and Shaw LM: Expression and function of the insulin receptor substrate proteins in cancer. Cel Commun Signal 7: 14, 2009

12. Taniguchi CM, Emanuelli B and Kahn CR: Critical nodes in signalling pathways: Insights into insulin action. Nat Rev Mol Cell Biol 7: 85-96, 2006.

13. Chan SH, Kikkawa U, Matsuzaki H, Chen JH and Chang WC: Insulin receptor substrate-1 prevents autophagy-dependen cell death caused by oxidative stress in mouse NIH/3T3 cells. J Biomed Sci 19: 64, 2012.

14. Bergmann U, Funatomi H, Kornmann M, Beger HG and Korc M: Increased expression of insulin receptor substrate-1 in human pancreatic cancer. Biochem Biophys Res Commun 220: 886-890, 1996.

15. Shi B, Sepp-Lorenzino L, Prisco M, Linsley P, deAngelis T and Baserga R: Micro RNA 145 targets the insulin receptor substrate-1 and inhibits the growth of colon cancer cells. J Biol Chem 282: 32582-32590, 2007.
16. Wu X, Cui CL, Chen WL, Fu ZY, Cui XY and Gong X: miR-144 suppresses the growth and metastasis of laryngeal squamous cell carcinoma by targeting IRS1. Am J Transl Res 8: 1-11, 2016.

17. Gao $\mathrm{C}$ and Chen YG: Dishevelled: The hub of Wnt signaling. Cell Signal 22: 717-727, 2010

18. Zhang Y, Wang F, Han L, Wu Y, Li S, Yang X, Wang Y, Ren F, Zhai Y, Wang D, et al: GABARAPL1 negatively regulates Wnt/ $\beta$-catenin signaling by mediating Dvl2 degradation through the autophagy pathway. Cell Physiol Biochem 27: 503-512, 2011.

19. Gao C, Cao W, Bao L, Zuo W, Xie G, Cai T, Fu W, Zhang J, Wu W, Zhang X, et al: Autophagy negatively regulates Wnt signalling by promoting Dishevelled degradation. Nat Cell Biol 12: 781-790, 2010.

20. Geng Y, Ju Y, Ren F, Qiu Y, Tomita Y, Tomoeda M, Kishida M, Wang Y, Jin L, Su F, et al: Insulin receptor substrate $1 / 2($ IRS1/2) regulates $\mathrm{Wnt} / \beta$-catenin signaling through blocking autophagic degradation of dishevelled2. J Biol Chem 289: 11230-11241, 2014.

21. Livak KJ and Schmittgen TD: Analysis of relative gene expression data using real-time quantitative PCR and the 2(-Delta Delta C(T)) method. Methods 25: 402-408, 2001.

22. Tan G, Tang X and Tang F: The role of microRNAs in nasopharyngeal carcinoma. Tumour Biol 36: 69-79, 2015.

23. Qiu F, Sun R, Deng N, Guo T, Cao Y, Yu Y, Wang X, Zou B, Zhang S, Jing T, et al: miR-29a/b enhances cell migration and invasion in nasopharyngeal carcinoma progression by regulating SPARC and COL3A1 gene expression. PLoS One 10: e0120969, 2015.

24. Verhoeven RJ, Tong S, Zhang G, Zong J, Chen Y, Jin DY, Chen MR, Pan $J$ and Chen $\mathrm{H}$ : NF- $\mathrm{KB}$ signaling regulates expression of Epstein-Barr virus BART MicroRNAs and long noncoding RNAs in nasopharyngeal carcinoma. J Virol 90: 6475-6488, 2016

25. Wang S, Zhang R, Claret FX and Yang H: Involvement of microRNA-24 and DNA methylation in resistance of nasopharyngeal carcinoma to ionizing radiation. Mol Cancer Ther 13: 3163-3174, 2014.

26. Carew RM, Browne MB, Hickey FB and Brazil DP: Insulin receptor substrate 2 and FoxO3a signalling are involved in E-cadherin expression and transforming growth factor- $\beta 1$-induced repression in kidney epithelial cells. FEBS J 278: 3370-3380, 2011.

27. Sorokin AV and Chen J: MEMO1, a new IRS1-interacting protein, induces epithelial-mesenchymal transition in mammary epithelial cells. Oncogene 32: 3130-3138, 2013.

28. Dearth RK, Cui X, Kim HJ, Kuiatse I, Lawrence NA, Zhang X, Divisova J, Britton OL, Mohsin S, Allred DC, et al: Mammary tumorigenesis and metastasis caused by overexpression of insulin receptor substrate 1 (IRS-1) or IRS-2. Mol Cell Biol 26: 9302-9314, 2006

29. Karolina DS, Armugam A, Tavintharan S, Wong MT, Lim SC, Sum CF and Jeyaseelan K: MicroRNA 144 impairs insulin signaling by inhibiting the expression of insulin receptor substrate 1 in type 2 diabetes mellitus. PLoS One 6: e22839, 2011.

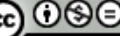

This work is licensed under a Creative Commons Attribution-NonCommercial-NoDerivatives 4.0 International (CC BY-NC-ND 4.0) License. 